\title{
PROCEDIMIENTO DE EVALUACIÓN DE EDIFICIOS DE CONCRETO REFORZADO BASADO EN DESEMPEÑO: DESARROLLO Y VALIDACIÓN
}

\author{
Mabel Mendoza Pérez ${ }^{(1)}$ y A. Gustavo Ayala Milián ${ }^{(2)}$
}

\begin{abstract}
RESUMEN
Se presenta un método para evaluar sísmicamente edificios de concreto reforzado, fundamentado en conceptos de ingeniería basada en desempeño; en la validez de la curva de capacidad como una propiedad del sistema estructural; y en la consideración de energía disipada por histéresis, introducida en el procedimiento mediante tasas de amortiguamiento equivalente en un sistema simplificado de un grado de libertad. El método permite evaluar de manera directa el desempeño de estructuras sometidas a demandas sísmicas crecientes ya que conduce a una curva de capacidad que aproxima satisfactoriamente la correspondiente derivada de análisis dinámicos incrementales.
\end{abstract}

Palabras claves: evaluación; desempeño; curva de capacidad; amortiguamiento

\begin{abstract}
This paper presents a seismic performance evaluation method for reinforced concrete buildings, based on concepts of performance-based engineering; the validity of the capacity curve as a property of the structural system; and on the consideration the energy dissipation due to hysteresis, included in the method by equivalent damping ratios on simplified degree of freedom system. The method allows in a direct way the performance evaluation of structures subjected to demands of increasing intensities since this leads to the capacity curve of a structure that approximates satisfactorily the capacity curve that derived from incremental dynamic analyses.
\end{abstract}

Key Words: evaluation; performance; capacity curve; damping

\section{INTRODUCCIÓN}

Las consecuencias destructivas de sismos recientes, han motivado la revisión de los procedimientos de evaluación para estructuras existentes y el desarrollo de procedimientos de diseño para nuevas construcciones que enfaticen conceptos de la filosofía de diseño sísmico por desempeño. Entre los procedimientos aproximados de evaluación sísmica de mayor difusión están el método del espectro de capacidad, el método de coeficientes de desplazamientos, el método $N 2$ y otros, referenciados en documentos como el FEMA 440 (ATC, 2005). Estos procedimientos tienen en común utilizar la curva de capacidad de una estructura, en la cual se representa la variación de una medida global del desempeño sísmico $v s$ diferentes intensidades de la demanda sísmica (e.g., desplazamiento máximo de azotea de un

Artículo recibido el 16 de julio de 2011 y aceptado para publicación el 24 de octubre de 2012

(1) Profesora Asociada, Departamento de Ingeniería Civil, División de Ingenierías, Campus Guanajuato, Universidad de Guanajuato; Av. Juárez No. 77, Col. Centro, C.P. 36000, Guanajuato, Gto.; mabel.mndz@ugto.mx

(2) Profesor Investigador, Coordinación de Mecánica Aplicada, Instituto de Ingeniería, Universidad Nacional Autónoma de México; Circuito Escolar s/n, Ciudad Universitaria, Delegación Coyoacán, C.P. 04510, México D.F.; gayalam@iingen.unam.mx 
edificio vs su cortante basal). La definición de esta curva como la propiedad estructural que caracteriza el desempeño sísmico de una estructura ante una demanda sísmica con características definidas, justifica la importancia de contar con procedimientos de análisis que la aproximen satisfactoriamente.

Para construir una curva de capacidad existen diversos métodos simplificados aproximados basados en fuerzas o desplazamiento. Los métodos basados en desplazamientos son los que representan de manera más realista las características y los efectos de la demanda sísmica sobre las estructuras, entre ellos destacan los propuestos por Aydinogliu (2003), Antoniu y Pinho (2004) y Alba (2005). Sin embargo, el único método para obtener una curva de capacidad "exacta" para una demanda sísmica dada, se basa en la aplicación de análisis dinámicos incrementales.

El análisis dinámico incremental (IDA, por sus siglas en inglés) es un método de análisis paramétrico que estima de manera "exacta" el comportamiento de una estructura bajo carga sísmica (Vamvatsikos y Cornell, 2002). Consiste en aplicar una serie de análisis no-lineales paso a paso a una estructura, usando como demanda uno o varios registros sísmicos, cada uno escalado a diferentes niveles de intensidad. De estos análisis se obtienen curvas IDA (figura 1a), en las cuales se representan índices de desempeño dependientes de niveles de intensidad de demanda (e.g. distorsiones máximas de entrepiso vs aceleraciones espectrales); y curvas de capacidad dinámica, donde el comportamiento de la estructura se representa en el espacio cortante basal vs desplazamiento máximo de azotea (Fragiadakis y Vamvatsikos, 2011), figura $1 b$.

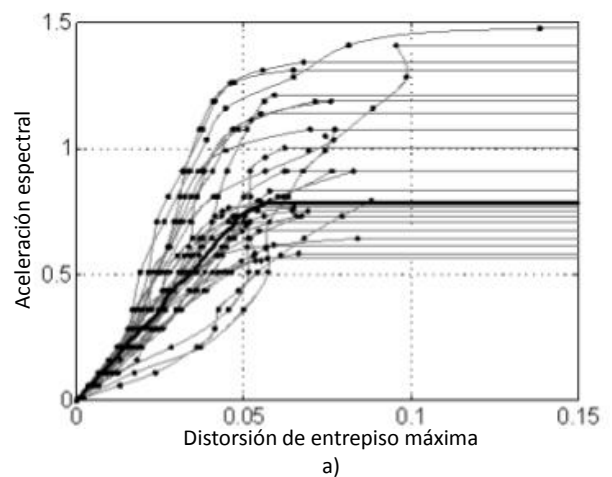

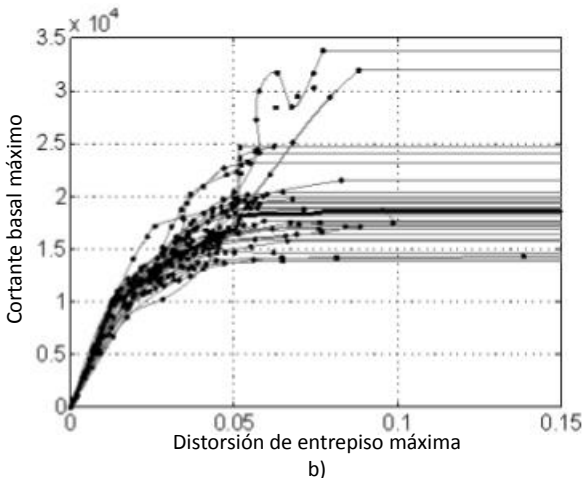

Figura 1. Representación de información obtenida de análisis IDA: (a) curvas IDA y (b) curvas de capacidad dinámica (Fragiadakis y Vamvatsikos, 2011)

Con los procedimientos simplificados de análisis basados en desplazamientos existentes, es posible obtener curvas de capacidad comparables en forma con la curva de capacidad dinámica para un registro sísmico particular; sin embargo, estas curvas tienen la limitante de que los desplazamientos asociados con los diferentes niveles de intensidad de demanda no corresponden a los de la curva de capacidad dinámica. Esta diferencia se debe a que en el cálculo aproximado de los desplazamientos no se considera la disipación de energía que ocurre debido al comportamiento histerético de los elementos estructurales cuando incursionan bajo excitación sísmica en el rango no-lineal de comportamiento. Para salvar esta limitante, se propone un método de evaluación sísmica que considera de manera aproximada la disipación de energía, mediante la inclusión iterativa de tasas de amortiguamiento equivalente en un sistema equivalente de un grado de libertad, correspondiente a los niveles de disipación de energía histerética del sistema estructural. De esta forma, la curva de capacidad calculada con el método propuesto conduce a desempeños aproximadamente iguales a los que se obtendrían de un análisis no-lineal paso a paso. 


\section{PROCEDIMIENTO PROPUESTO}

El método de evaluación simplificado que se presenta, tiene sus bases en la formulación desarrollada por Requena y Ayala (2000) para evaluar el comportamiento no-lineal de marcos planos; en la que se incluyó el efecto de la variación de la distribución de cargas inducidas por sismo, debido al cambio de las características estructurales relacionadas con el comportamiento inelástico de la estructura, el efecto de acumulación de daño y de los modos superiores de vibrar.

En el método propuesto, la evaluación de la estructural se realiza mediante el cálculo de una curva de capacidad construida a partir de los resultados de una serie de análisis modales espectrales evolutivos (AMEE); cada uno correspondiente a un incremento de la demanda sísmica y asociado con un nivel de daño producido. Estrictamente, en su aplicación es necesario realizar tantos análisis como niveles de daño (comúnmente representados por la aparición de articulaciones plásticas) ocurran antes de que la estructura alcance su desplazamiento objetivo. Sin embargo, para fines de aplicación práctica, no es necesario realizar un análisis cada vez que un elemento alcance su capacidad elástica, ya que una aproximación aceptable es realizar cada análisis (nuevo punto de la curva de capacidad) cada vez que se tiene un conjunto de secciones de elementos en las que aproximadamente ocurra la fluencia bajo un mismo nivel de demanda. Esta simplificación permite realizar un número reducido de análisis y utilizar herramientas de análisis estructural disponibles a profesionales de la ingeniería estructural, e.g., SAP2000 (CSI, 2000); en ella, se considera que el daño inducido por sismo se concentra en las secciones extremas de los elementos estructurales y se modela de manera simplista al introducir una rótula de rigidez nula.

El procedimiento de análisis se compone de dos etapas: en la primera se define la demanda sísmica que corresponda al nivel de diseño para el cual se requiere evaluar la estructura y las propiedades de los elementos estructurales; y en la segunda se realiza la construcción de la curva de capacidad y evaluación sísmica de la estructura para distintas intensidades de la demanda.

\section{Primera etapa}

i. Definir la demanda sísmica de acuerdo con el nivel de diseño para el cual se evaluará la estructura. Lo anterior se logra mediante espectros de diseño suavizados o espectros de peligro uniforme para un sitio particular.

ii. Determinar los diagramas momento-curvatura de los elementos estructurales (vigas y columnas), de dónde se obtendrán los momentos de fluencia $\left(M_{y}\right)$ de cada sección.

\section{Segunda etapa}

iii. Identificar la aparición del primer daño en la estructura (figura 2). Para realizar este paso se define un modelo sin daño de la estructura, el cual se analiza bajo la acción de cargas verticales y un análisis modal espectral con la demanda sísmica completa donde se considera un porcentaje de amortiguamiento del $5 \%$, correspondiente al valor aceptado de amortiguamiento viscoso para edificios de concreto reforzado $\left(\xi_{o}\right)$. Con los resultados obtenidos, se determina el nivel de demanda sísmica necesario para que aparezca la primera fluencia en la estructura mediante el cálculo del menor factor de escala que se obtiene para los extremos de cada elemento. En este paso el factor de escala $\left(S f_{i=1}\right)$ es el cociente de la diferencia del momento de fluencia de la sección $\left(M_{y}\right)$ y el momento actuante $\left(M_{a c(i=l)}\right)$ que para este paso es el momento derivado del análisis de carga vertical $\left(M_{c v}\right)$, entre el momento derivado del análisis modal espectral evolutivo $\left(M_{\text {int }(i=1)}\right)$. 


$$
S f_{i=1}=\frac{M_{y}-M_{a c(i=1)}}{M_{\operatorname{int}(i=1)}}=\frac{M_{y}-M_{c v}}{M_{\operatorname{int}(i=1)}}
$$

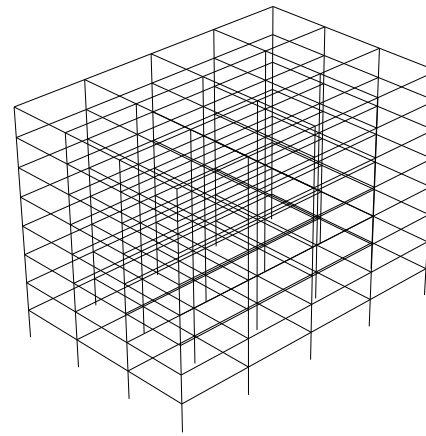

Modelo sin daño

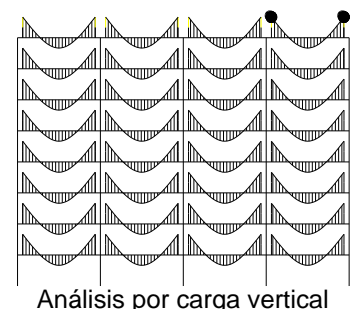

Análisis por carga vertical $M_{a c(i=1)}=M_{c v}$

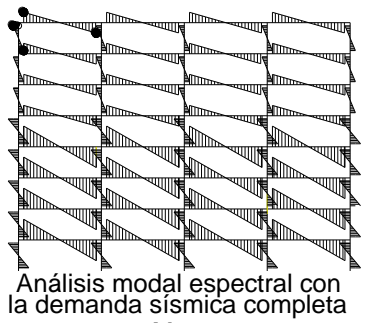
$M_{\text {int }}(i=1)$

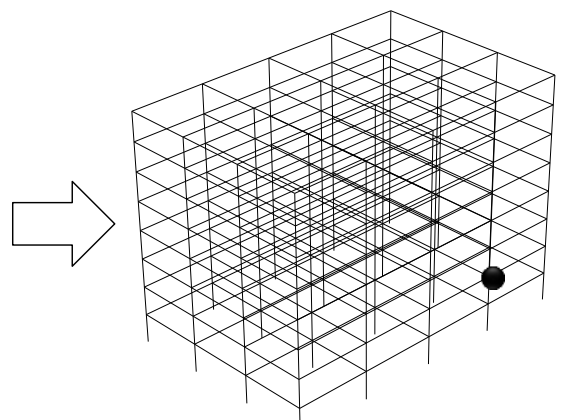

Se presenta el primer daño $S f_{i=1}$

Figura 2. Aparición del primer daño en la estructura

iv. Definir a partir de los resultados del punto anterior el punto de fluencia (figura 3 ) de la curva de capacidad. Las coordenadas que definen éste punto se obtienen con las ecuaciones 2 a 5 ; donde $V_{i=1}$ es el cortante basal, $\delta_{i=1}$ es el desplazamiento, $M_{i=1}$ es el momento torsor y $\theta_{i=1}$ es la rotación, del punto de fluencia de la curva de capacidad; y a su vez $V e_{i=l}$ es el cortante basal, $M e_{i=1}$ es el momento torsor, $\delta e_{i=1} \mathrm{y}$ $\theta e_{i=1}$ son el desplazamiento y la rotación del punto de control respectivamente, correspondientes al primer análisis modal espectral evolutivo.

$V_{i=1}=\left(V e_{i=1}\right)\left(S f_{i=1}\right)$

$\delta_{i=1}=\left(\delta e_{i=1}\right)\left(S f_{i=1}\right)$

$M_{i=1}=\left(M e_{i=1}\right)\left(S f_{i=1}\right)$

$\theta_{i=1}=\left(\theta e_{i=1}\right)\left(S f_{i=1}\right)$

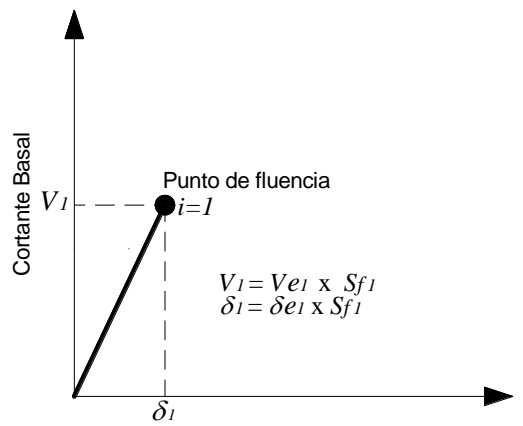

Figura 3. Punto de fluencia de una curva de capacidad 
v. Calcular el incremento de demanda que define el nivel de daño sucesivo asignado a la estructura (ecuación 6), donde el factor de escala $\left(S f_{i+1}\right)$ es el cociente de la diferencia entre el momento de fluencia de la sección $\left(M_{y}\right)$ y el momento actuante $\left(M_{a c(i+1)}\right)$ definido a partir de este punto por la ecuación 7 y el momento derivado del análisis modal espectral evolutivo $\left(M_{i n t}(i+1)\right.$. Este paso estrictamente se realiza tantas veces como articulaciones plásticas ocurran antes de que la estructura alcance su desplazamiento objetivo, sin embargo, para hacer práctico el método, se recomienda trabajar con rigideces asociadas a niveles de daño correspondientes a grupos de articulaciones plásticas que se estima ocurrirán durante etapa. No obstante, estimar el número y localización de nuevas articulaciones por grupo para cada análisis es un problema no-trivial, ya que errores en esta estimación pueden subestimar o sobrestimar la rigidez de la estructura en esta etapa de análisis. Al final de esta sección se propone el uso de un método aproximado para definir la distribución de daño y la correspondiente rigidez que mejor represente la condición estructural efectiva en esta etapa. Es importante mencionar que el incremento de desplazamiento correspondiente a esta etapa de análisis no considera la disipación de energía por histéresis, por lo que es necesario efectuar una corrección en la que si se considera.

$S f_{i+1}=\frac{M_{y}-M_{a c(i+1)}}{M_{i n t(i+1)}}$
$M_{a c(i+1)}=M_{a c(i-1)}+\left(M_{i n t(i-1)}\right)\left(S f_{i-1}\right)$

vi. Determinar los incrementos (figura 4) correspondientes al cortante basal $\left(\Delta V_{i}\right)$, desplazamiento máximo $\left(\Delta \delta_{i}\right)$, momento torsor $\left(\Delta M_{i}\right)$ y rotación del punto de control $\left(\Delta \theta_{i}\right)$, con las ecuaciones 8 a 11 . Estos incrementos corresponden al $i$-ésimo análisis modal espectral evolutivo y definen el punto $(i+1)$ de la curva de capacidad (punto no corregido).

$$
\begin{aligned}
\Delta V_{i} & =\left(V e_{i+1}\right)\left(S f_{i+1}\right) \\
\Delta \delta_{i} & =\left(\delta e_{i+1}\right)\left(S f_{i+1}\right) \\
\Delta M_{i} & =\left(M e_{i+1}\right)\left(S f_{i+1}\right) \\
\Delta \theta_{i} & =\left(\theta e_{i+1}\right)\left(S f_{i+1}\right)
\end{aligned}
$$

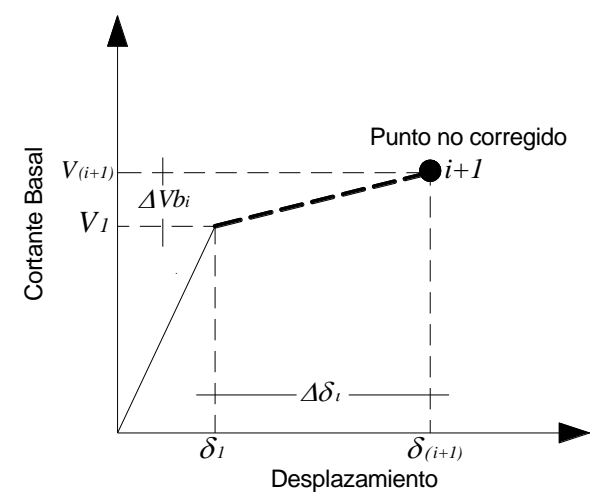

Figura 4. Punto no corregido de la curva de capacidad

vii. Considerar la disipación de energía por histéresis para corregir las demandas de desplazamiento y rotaciones calculadas en el paso anterior, que definen el punto corregido $(i+1)$ de la curva de capacidad (figura 5). Esto se realiza al analizar de manera iterativa un sistema equivalente de un grado de libertad 
(figura 6) con comportamiento definido por la curva de capacidad y tazas de amortiguamiento equivalente $\left(\xi_{\text {eq }}\right)$ como se indica en la ecuación 12; donde $\xi_{\text {hist }}$ es la fracción de amortiguamiento crítico correspondiente a la disipación de energía por histéresis y $\xi_{o}$ es la fracción del amortiguamiento viscoso de la estructura (5\%). Para aproximar $\xi_{\text {hist }}$ se utiliza la ecuación 13 (Rosenblueth y Herrera, 1964); donde $\alpha$ es la relación de rigidez de post fluencia a rigidez inicial y $\mu$ es la ductilidad asociada al desplazamiento máximo de cada análisis. Ya que durante la respuesta del oscilador el desplazamiento máximo ocurre un número reducido de veces se consideró que un valor aceptable para estimar el $\xi_{\text {hist }}$ es considerar el amortiguamiento asociado con dos tercios del desplazamiento máximo. Como resultado se tiene un porcentaje de $\xi_{e q}$ que se incluye en el sistema de un grado de libertad para obtener incrementos reducidos de $\Delta V_{i}, \Delta \delta_{i}, \Delta M_{i}$ y $\Delta \theta_{i}$. Lo anterior es un proceso iterativo, donde se verifica el porcentaje de error entre las reducciones de incrementos calculados $(e)$ en cada iteración; si la diferencia es del 5\% o menor, se procede a designar el siguiente nivel de daño en la estructura (punto $v$ ); en caso contrario, se realiza un nuevo cálculo de porcentaje de $\xi_{e q}$ acumulable al porcentaje anterior, para tener una nueva reducción en $\Delta V_{i}, \Delta \delta_{i}, \Delta M_{i}$ y $\Delta \theta_{i}$. Es importante hacer notar que al considerar la disipación de energía por histéresis, los niveles de daño originalmente establecidos cambian, haciendo necesario considerarlos al definir el nuevo nivel de demanda.

$$
\begin{aligned}
& \xi_{\text {eq(i) }}=\xi_{\text {hist }(i)}+\xi_{o} \\
& \xi_{\text {hist }}=\frac{2}{\pi}\left[\frac{(1-\alpha)(\mu-1)}{\mu-\alpha \mu+\alpha \mu^{2}}\right]
\end{aligned}
$$

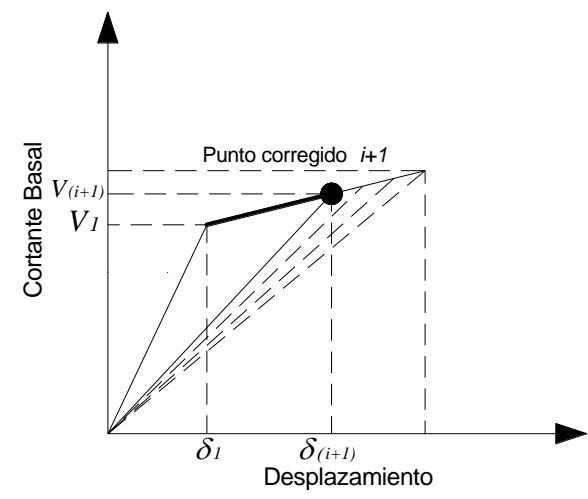

Figura 5. Punto corregido de la curva de capacidad

viii. Construir la rama de la curva de capacidad que representan el comportamiento inelástico de la estructura. Una vez que se consideró la disipación de energía por histéresis mediante una fracción de amortiguamiento adicional al viscoso y se obtuvieron los incrementos reducidos de $\Delta V_{i}, \Delta \delta_{i}, \Delta M_{i}$ y $\Delta \theta_{i}$, se definen las coordenadas de la rama $i$-ésima de la curva de capacidad, con las ecuaciones 14 a 17.

$$
\begin{aligned}
& V_{i+1}=V_{1}+\sum \Delta V_{i} \\
& \delta_{i+1}=\delta_{1}+\sum \Delta \delta_{i} \\
& M_{i+1}=M_{1}+\sum \Delta M_{i} \\
& \theta_{i+1}=\theta_{1}+\sum \Delta \theta_{i}
\end{aligned}
$$




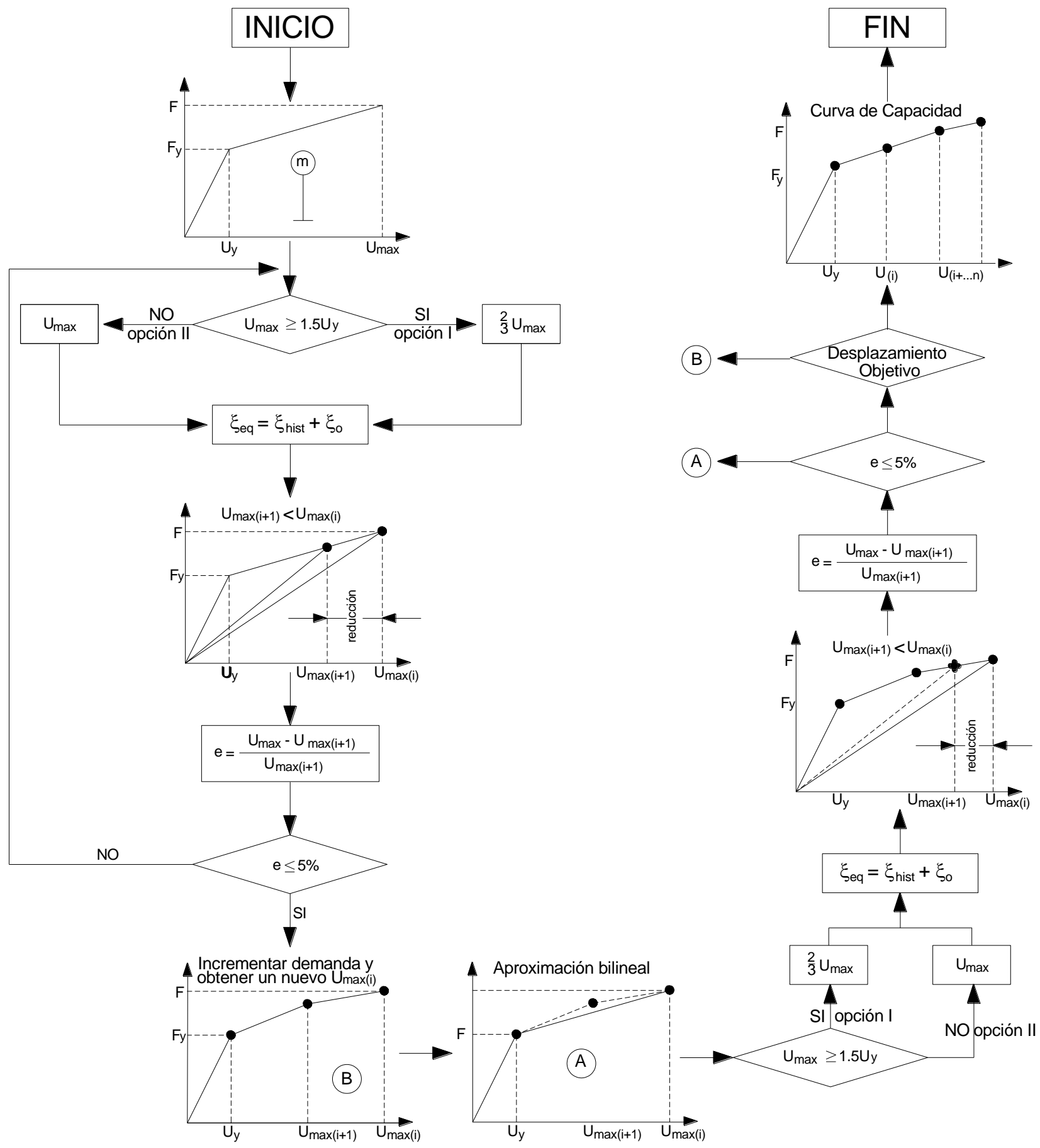

Figura 6. Algoritmo de corrección de demanda de desplazamiento y rotaciones

ix. Evaluar el desempeño de la estructura. A partir de los resultados con los que se construyó la curva de capacidad de la estructura se puede determinar su desempeño ante una demanda sísmica particular sin necesidad de realizar acciones complementarias, como serían las que involucran la aplicación de algún método de evaluación adicional (método del espectro de capacidad, N2, método de los coeficientes, entre otros). Esto es posible debido a que la curva de capacidad construida representa la relación entre 
intensidad de la demanda sísmica y el correspondiente desempeño de la estructura, en este caso desplazamientos de un punto de control.

Como se mencionó en el paso $v$ del método, estimar los niveles de daño asociados con una etapa particular de análisis es un problema complejo, por lo que en este artículo se recomienda para definir estos niveles de daño el uso de un método predictor-corrector en el que se use una configuración de daño intermedia en vez de la configuración inicial o final del incremento de intensidad de demanda sísmica, asociada a una rigidez secante en la etapa en vez de la tangente al inicio o final de la misma. Este proceder que involucra un análisis adicional por incremento de intensidad, mejora la precisión del método ya que acerca la curva de capacidad aproximada a la que se obtendría mediante análisis dinámicos incrementales. Con base en lo anterior para todas las ramas subsecuentes a la elástica (figura 7), se propone realizar un análisis predictor, primero con un incremento del $50 \%$ de la intensidad de demanda deseada para el punto $i+1$ de la curva de capacidad; de los resultados de éste, se identifican los elementos que alcanzan su fluencia y se define un modelo de la estructura. Una vez calculados los resultados correspondientes a este nivel de daño intermedio, se realiza un nuevo análisis con $100 \%$ del incremento de la intensidad de demanda deseada, para obtener las coordenadas que definen el punto $i+1$ de la curva de capacidad (figura 8) que debe corregirse como se indica en el punto vii del método (figura 9 y 10). Cabe mencionar que al incluir información previa de un nivel de daño relacionado con un nivel de intensidad de demanda, se logra una convergencia en la corrección del punto $i+l$ con un número menor de iteraciones.

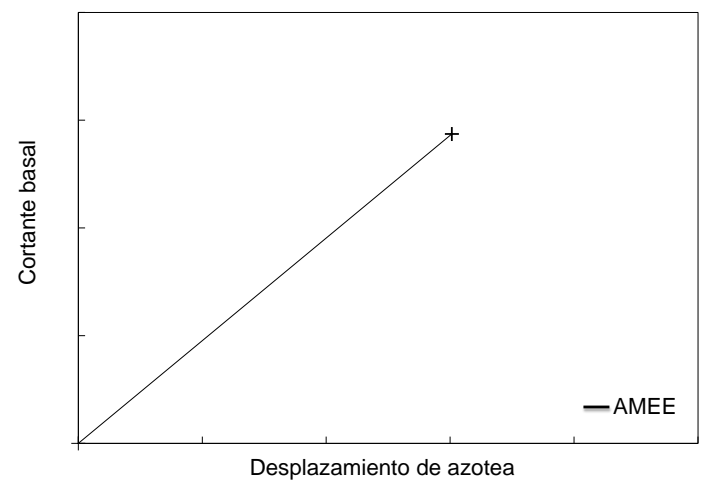

Figura 7. Rama elástica de la curva de capacidad

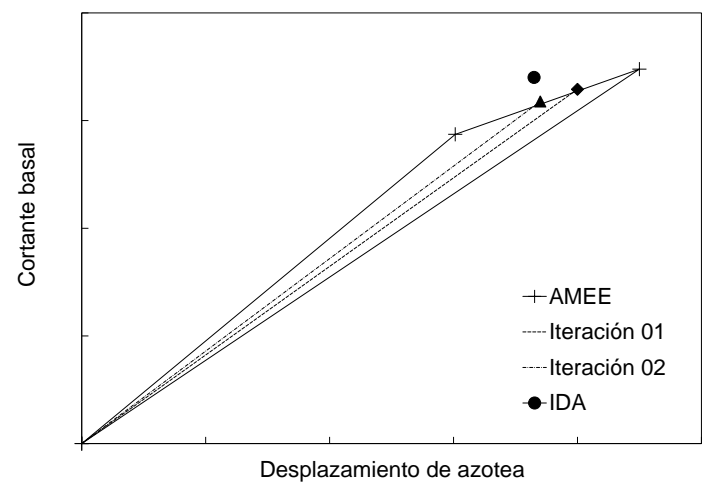

Figura 9. Corrección del punto i+1

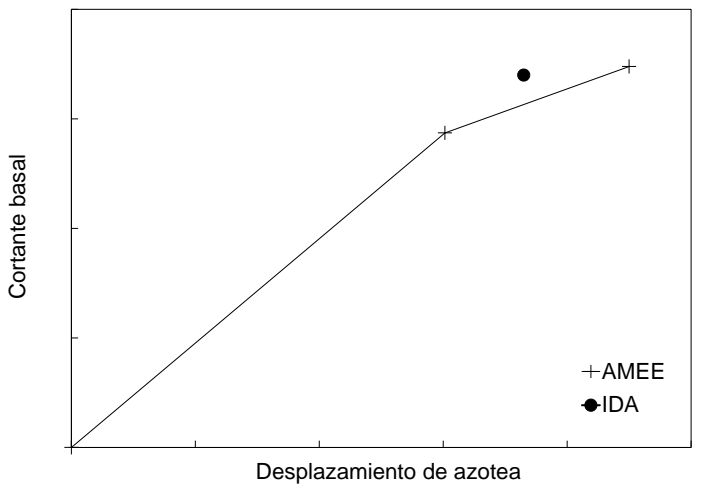

Figura 8. Punto i+1 sin corrección

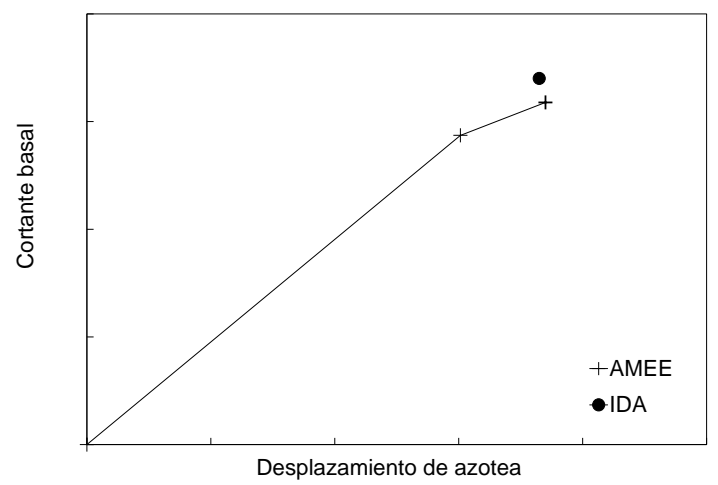

Figura 10. Punto i+1 corregido 


\section{EJEMPLO DE APLICACIÓN}

Para ilustrar la aplicación de este procedimiento se eligió un edificio de concreto reforzado de ocho niveles diseñado por Ortega (2001), que presenta igual excentricidad en masa en todos sus niveles. El edificio en estudio está formado por marcos de concreto reforzado; la planta tipo tiene cuatro crujías de $8 \mathrm{~m}$ en dirección $\mathrm{X}$, tres crujías de $7 \mathrm{~m}$ en dirección $\mathrm{Y}$ y cuenta con trabes secundarias en el sentido $\mathrm{X}$ al centro de cada crujía de $7 \mathrm{~m}$. El espesor de la losa se considera de $0.12 \mathrm{~m}$, la altura de entrepiso en todos los casos es de $3.3 \mathrm{~m}$. En la figura 11 se muestra la planta tipo del edificio, una vista tridimensional y los cortes en dirección X y Y. En la tabla 1 se muestra las dimensiones de los elementos estructurales.

En cuanto a las propiedades de los materiales se consideraron los siguientes valores nominales: concreto clase I con resistencia a la compresión de f'c $=250 \mathrm{~kg} / \mathrm{cm}^{2}$, módulo de elasticidad de $E c=221359 \mathrm{~kg} / \mathrm{cm}^{2}$ y peso volumétrico de $\mathrm{PV}=2400 \mathrm{~kg} / \mathrm{m}^{3}$; acero de refuerzo con un esfuerzo de fluencia de $f y=4200 \mathrm{~kg} / \mathrm{cm}^{2}$ y módulo de elasticidad de $E s=2 \times 10^{6} \mathrm{~kg} / \mathrm{cm}^{2}$. La demanda empleada en la evaluación del comportamiento sísmico para las dos direcciones principales de la planta fue la componente NS del sismo del 19 de septiembre de 1985 registrado en la estación de la Secretaría de Comunicaciones y Transportes, SCT, en la ciudad de México (i.e., señales 100\% correlacionadas). Para realizar los análisis modales espectrales evolutivos se utilizó como herramienta de análisis estructural comercial el programa SAP2000 (CSI, 2000) y para los análisis dinámicos incrementales el programa de análisis no-lineal CANNY-E (Li, 1996).

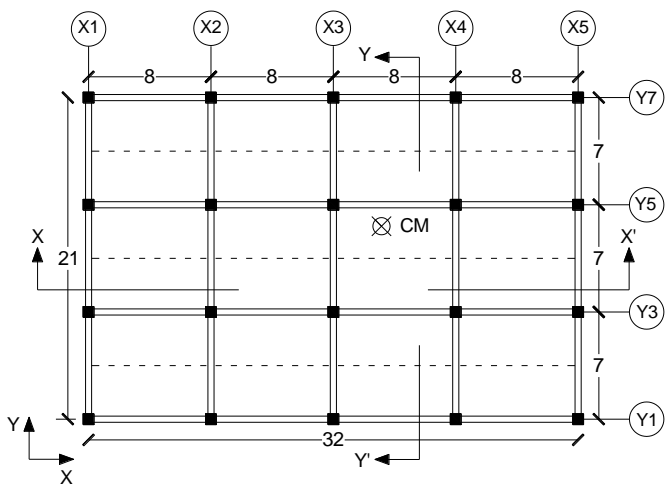

a)

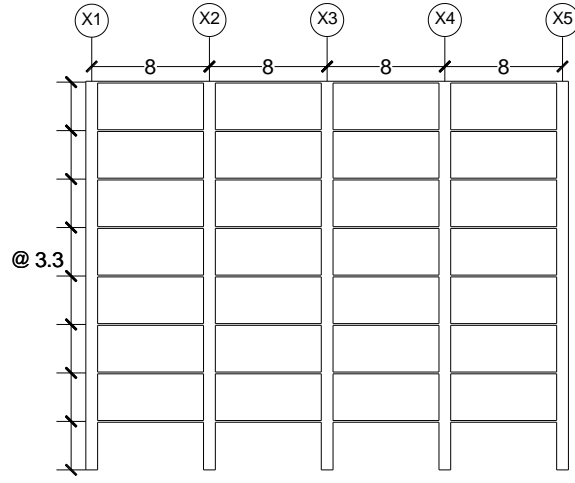

c)

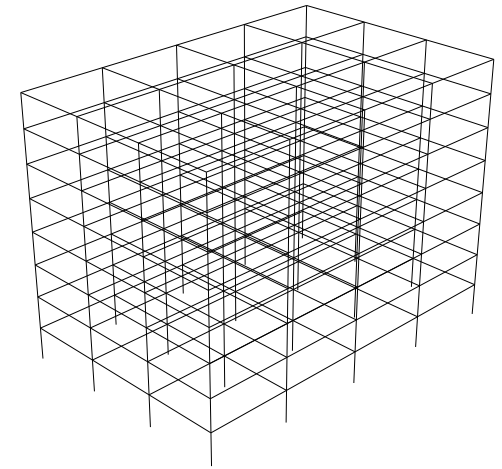

Acotación - m b)

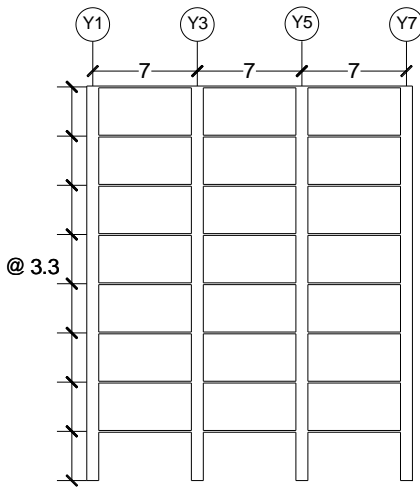

d)

Figura 11. (a) Planta tipo del edificio, (b) Vista tridimensional, (c) Corte en dirección X y (d) Corte en dirección Y 
Con el objetivo de evaluar el potencial del método propuesto se estudió la estructura de dos maneras diferentes: en la primera se estudió el comportamiento del marco longitudinal sobre el eje Y1 (8SF) y en la segunda el comportamiento de la estructura tridimensional (8SB). En la presentación y análisis de resultados se realizan comparaciones entre las curvas de capacidad construidas con el método propuesto (AMEE) y las correspondientes curvas de capacidad dinámicas. Es importante mencionar que para descartar la incertidumbre en los resultados al usar una regla de combinación modal en la aplicación de los AMEE, se utilizaron análisis modales en el tiempo. En las gráficas que a continuación se muestran, la letra $i$ se refiere a la intensidad de demanda sísmica.

Como primer paso en este estudio, se aplicó el AMEE sin considerar la influencia de la disipación de energía por histéresis, para evidenciar la no correspondencia de resultados con los obtenidos de un IDA (i.e., diferencias entre desplazamientos asociados con una misma intensidad de demanda sísmica). En la figura 12 se presenta una comparativa entre las curvas de capacidad obtenidas con el AMEE y el IDA, correspondientes al modelo 8SF; y en las figura 13 y 14 se muestran las curvas correspondientes al modelo 8SB en las direcciones $\mathrm{X}$ y $\mathrm{Y}$ respectivamente. En ambos casos se observa la falta de correspondencia de los desplazamientos asociados con una misma intensidad de demanda sísmica calculados con los dos procedimientos, y el efecto acumulativo de esas diferencias para el AMEE en cada paso del análisis, presentando diferencias mayores del $50 \%$.

Para corregir esta limitante y minimizar el error acumulado, en cada análisis AMEE se incluyen tasas de amortiguamiento equivalente propuestas, que consideran de manera aproximada la disipación de energía por histéresis en cada rama de la curva de capacidad, con lo que se pretende representar de manera más aproximada el comportamiento inelástico de la estructura.

Tabla 1. Dimensiones de elementos estructurales

\begin{tabular}{ccc}
\hline NIVEL & ELEMENTO ESTRUCTURAL & DIMENSIÓN $(\mathrm{m})$ \\
\hline $1-8$ & Columnas & $0.80 \times 0.80$ \\
$1-8$ & Vigas (ejes Y1, Y3, Y5 y Y7) & $0.40 \times 0.80$ \\
$1-8$ & Vigas (ejes Y2, Y4, y Y6) & $0.25 \times 0.60$ \\
\hline
\end{tabular}

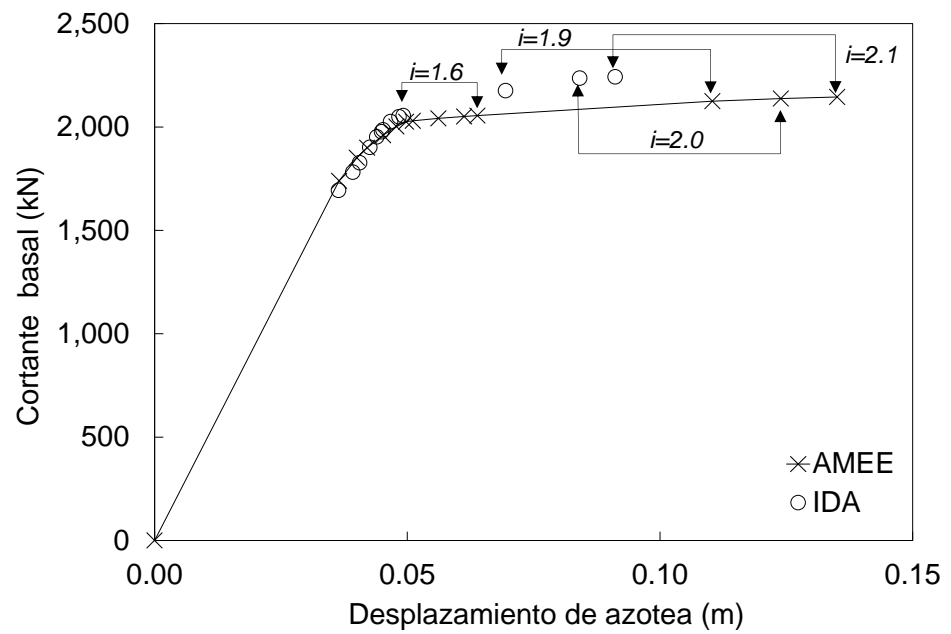

Figura 12. Curva de capacidad sin corrección del modelo 8SF 


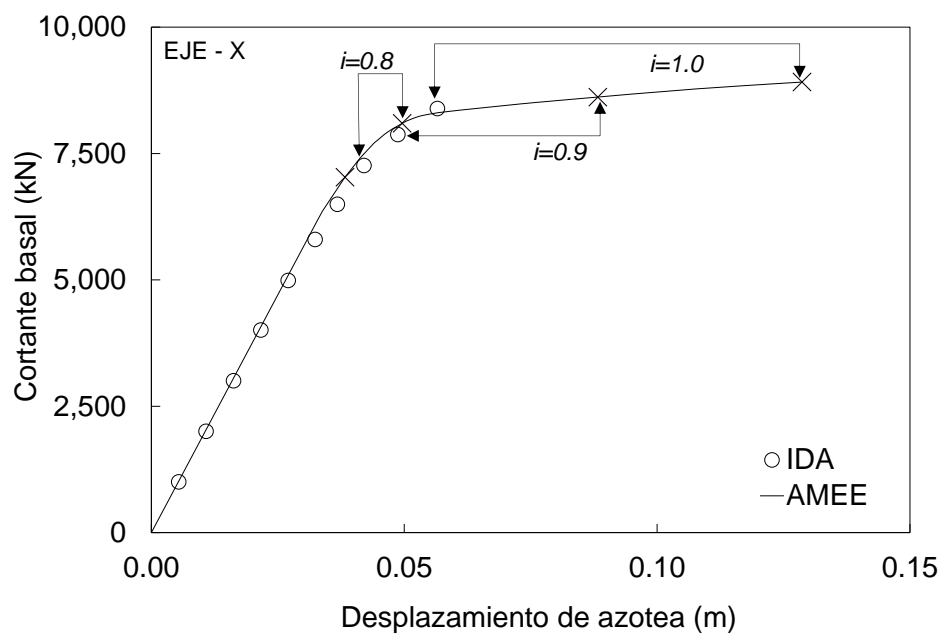

Figura 13. Curva de capacidad sin corrección del modelo 8SB (dirección X)

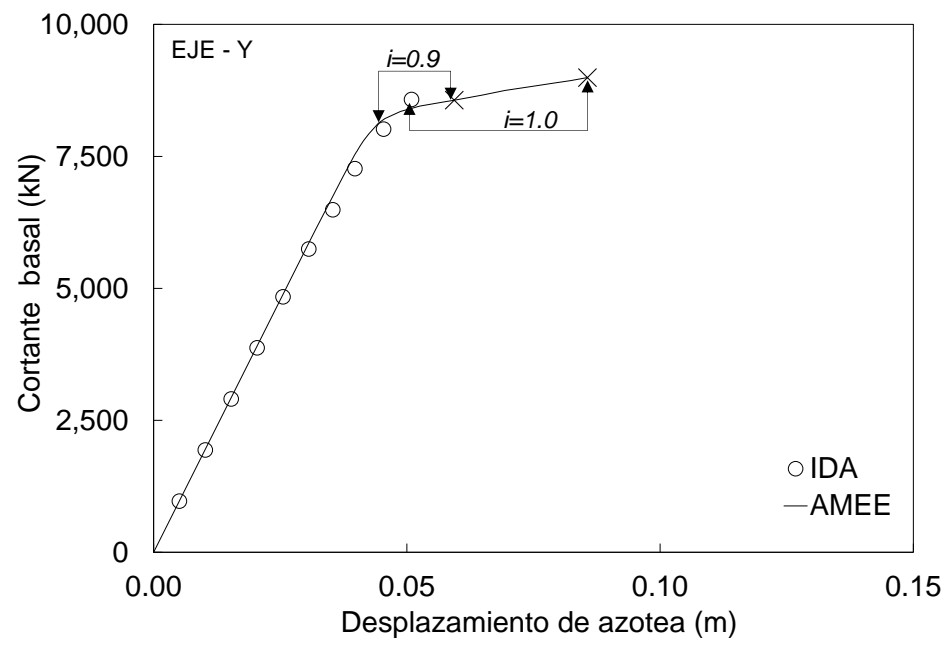

Figura 14. Curva de capacidad sin corrección del modelo 8SB (dirección Y)

En la evaluación del modelo $8 S F$ cada punto de la curva de capacidad (figura 15) corresponde a la aparición de daño en un conjunto de elementos que aproximadamente fluyen bajo un mismo nivel de demanda sísmica. Resultado del análisis de cargas verticales y el primer análisis modal en el tiempo, se encuentra que la intensidad de demanda necesaria para que aparezca la primera fluencia en éste modelo es igual $\mathrm{i}=1.21$; éste primer factor de escala define el punto de fluencia de la curva de capacidad, caracterizado por un desplazamiento de azotea de $0.036 \mathrm{~m}$ y un cortante basal de $1739.53 \mathrm{kN}$. En el intervalo de desempeño definido por las intensidades de demanda 1.21 a 1.50 , se presenta un daño aproximado del 50\% de los elementos estructurales; en este intervalo se observa una excelente aproximación, ya que las diferencias punto a punto entre los desplazamientos obtenidos con el AMEE y el correspondiente IDA no supera 1\%. Para intensidades de demanda sísmica mayores de 1.5 veces la original, se presentan mecanismos locales de inestabilidad y al llegar a una intensidad de demanda 2.1 veces mayor, los elementos estructurales dañados son más de la mitad (figura 16); en este intervalo de desempeño existe un error promedio del $5.9 \%$ en la correspondencia punto a punto entre los desplazamientos asociados con iguales intensidades de demanda sísmica que se obtienen con el AMEE y el IDA. En la figura 17 se muestra una comparación de las distorsiones de entrepiso y desplazamientos 
laterales para las intensidades límite de los intervalos de desempeño antes mencionados $(\mathrm{i}=1.21, \mathrm{i}=1.50$, $\mathrm{i}=2.10$ ), para tres métodos diferentes: AMEE no corregido, AMEE corregido y análisis dinámico incremental. Aquí se destaca la diferencia de magnitud entre los desplazamientos no corregidos y corregidos; y como estos últimos son comparables tanto en magnitud y forma con los que se obtienen de un análisis dinámico incremental.

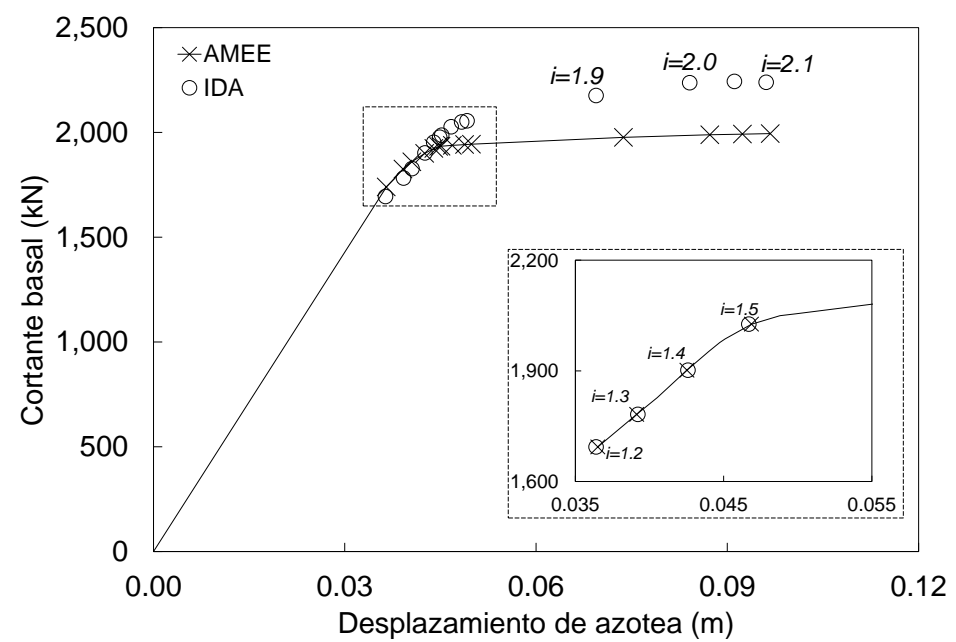

Figura 15. Curva de capacidad del modelo 8SF
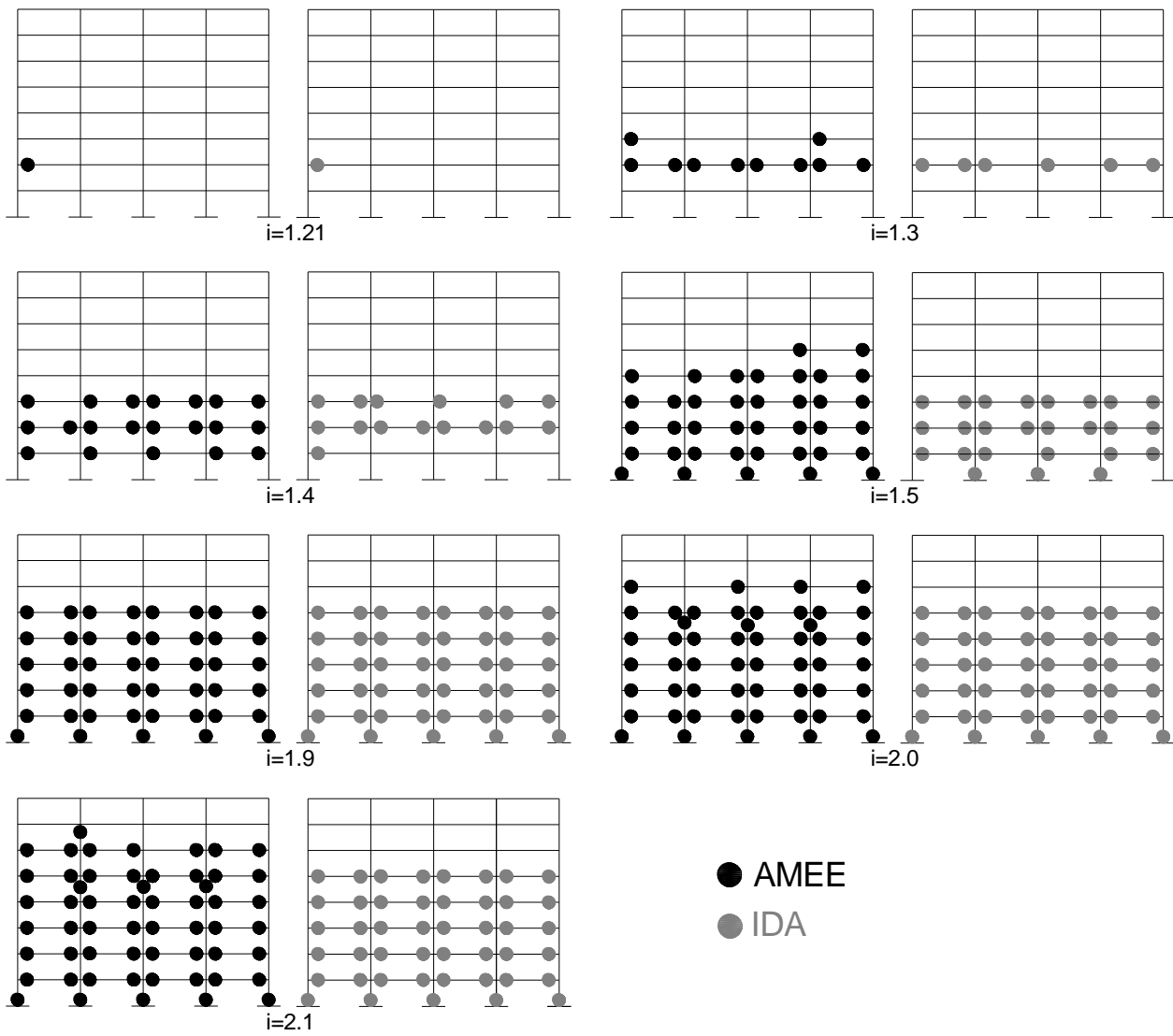

AMEE

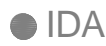

Figura 16. Daño del modelo 8SF a diferentes intensidades de demanda sísmica 
Intensidad de demánda sísmica $\mathrm{i}=1.21$
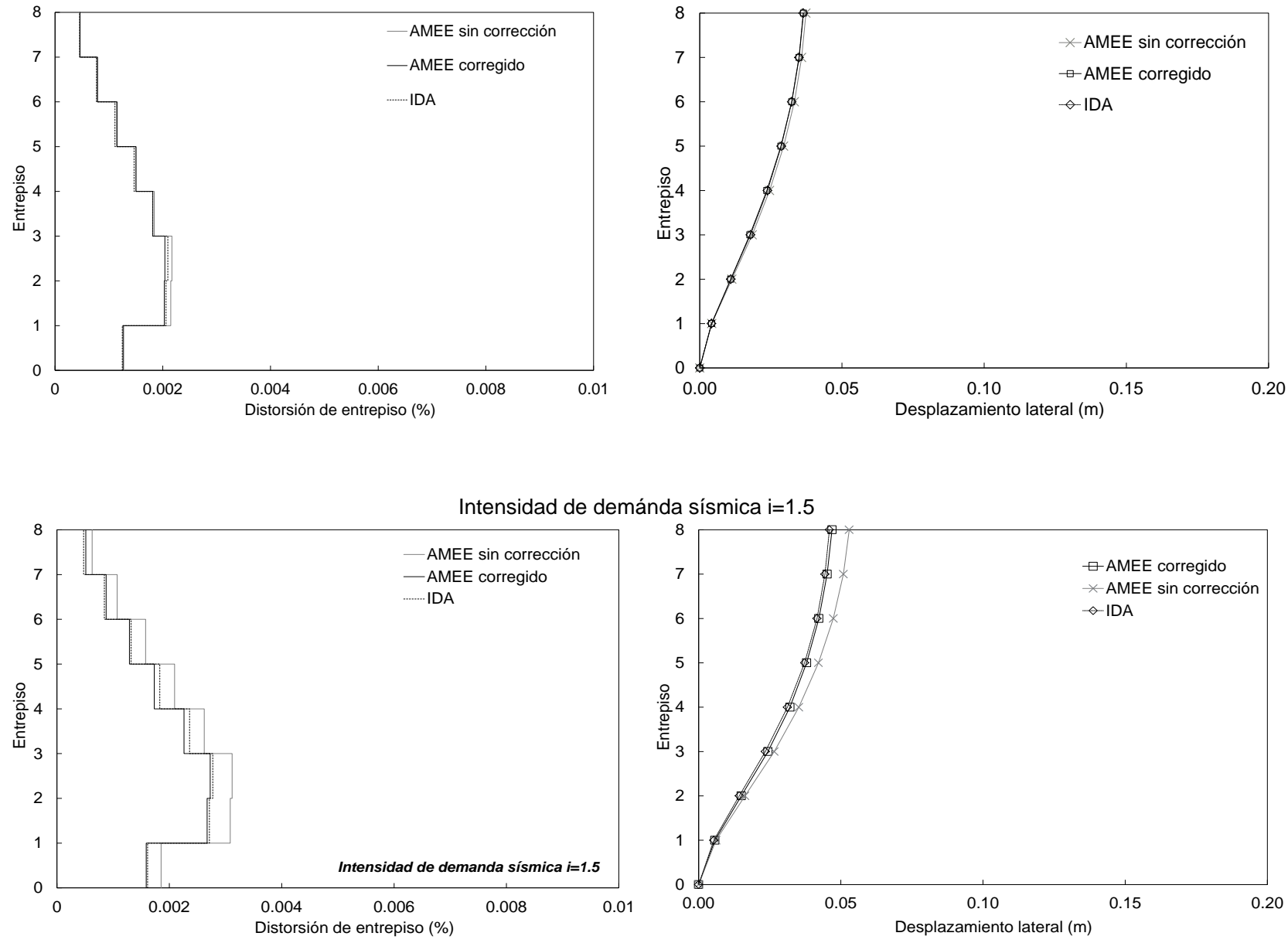

Intensidad de demánda sísmica $\mathrm{i}=2.1$

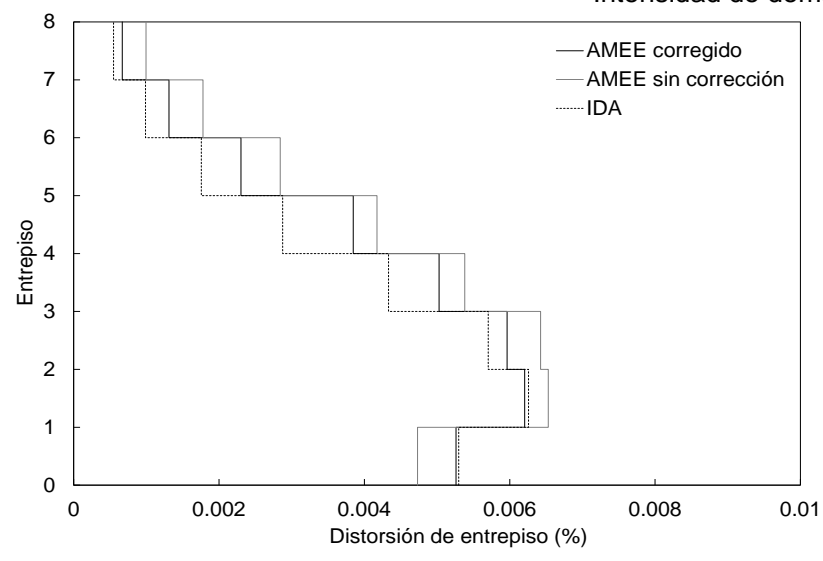

a)

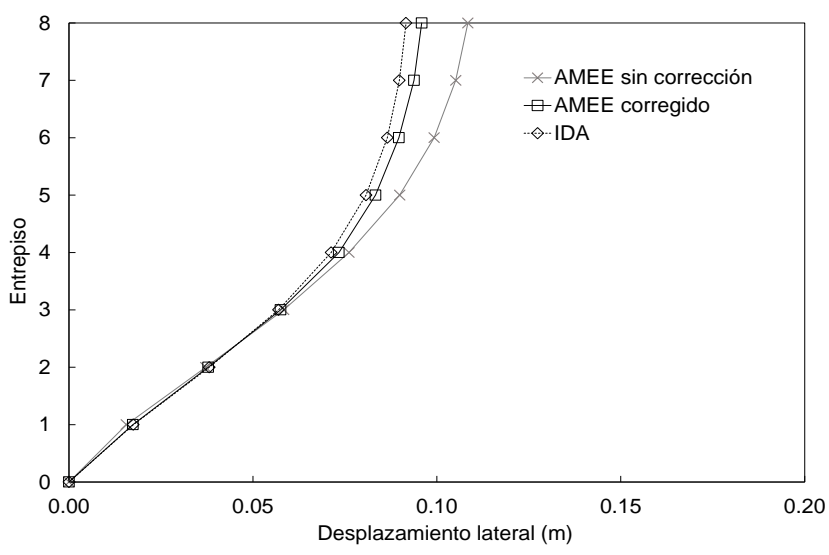

b)

Figura 17. Indicadores de desempeño global del modelo 8SF: (a) Distorsiones de entrepiso y (b) Desplazamientos laterales 
Para evaluar el comportamiento del modelo $8 S B$ de una manera práctica, se utilizó el algoritmo predictor-corrector para definir el nivel de daño a incluir en cada análisis modal en el tiempo. Debido a que el objetivo del estudio es validar el potencial del AMEE, para eliminar la incertidumbre que origina la correlación entre registros en dos direcciones ortogonales, se justifica el aplicar la misma componente de demanda sísmica en la dirección longitudinal y transversal, con porcentajes de intensidades iguales.

Para este modelo, el nivel de demanda necesario para que aparezca la primera fluencia es $59 \%$ de la intensidad total de demanda y define los puntos de fluencia de las curvas de capacidad (figura 18, $19 \mathrm{y}$ 20). El intervalo de desempeño delimitado por las intensidades de demanda 0.59 a 1.00 muestra la evolución de daño de aproximadamente $50 \%$ de los elementos estructurales (figura 21). En este intervalo la diferencia de desplazamiento punto a punto entre el AMEE y el IDA es del orden del $1 \%$ en las direcciones $\mathrm{X}$ y Y, mientras en la dirección rotacional se tiene 3\% de diferencia. Para intensidades de demanda mayores al 1.00 las diferencias entre los métodos incrementan: 2.4, 1.67 y 6.8\% para la dirección $\mathrm{X}, \mathrm{Y}$ y rotacional respectivamente. En las figuras 22 y 23 se muestran las distorsiones de entrepiso y desplazamientos laterales en la dirección $\mathrm{X}$ y $\mathrm{Y}$ respectivamente, aquí se observa como los desplazamientos obtenidos de un AMEE corregido y un IDA son comparables en magnitud y forma.

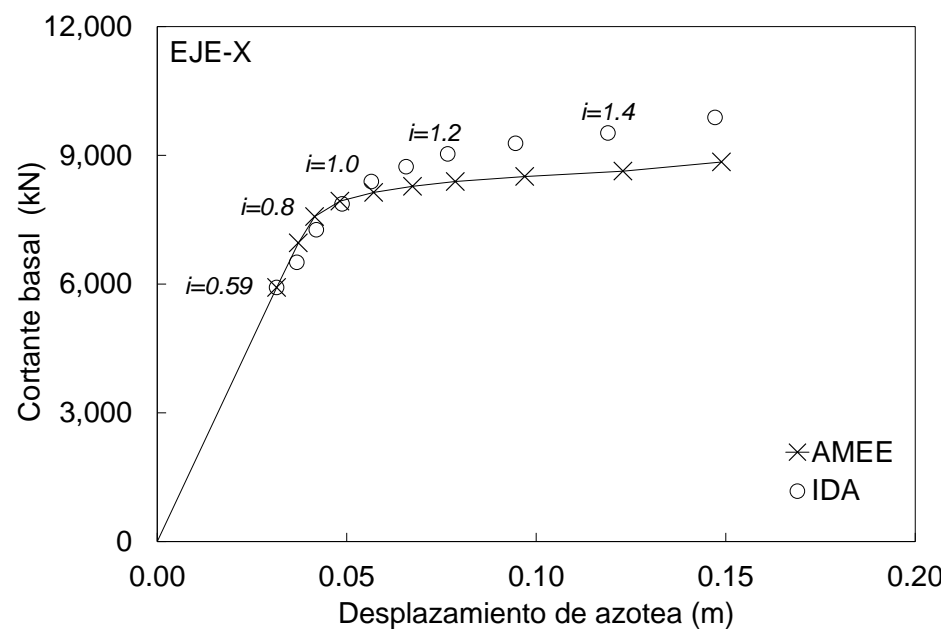

Figura 18. Curva de capacidad del modelo 8SB (dirección X)

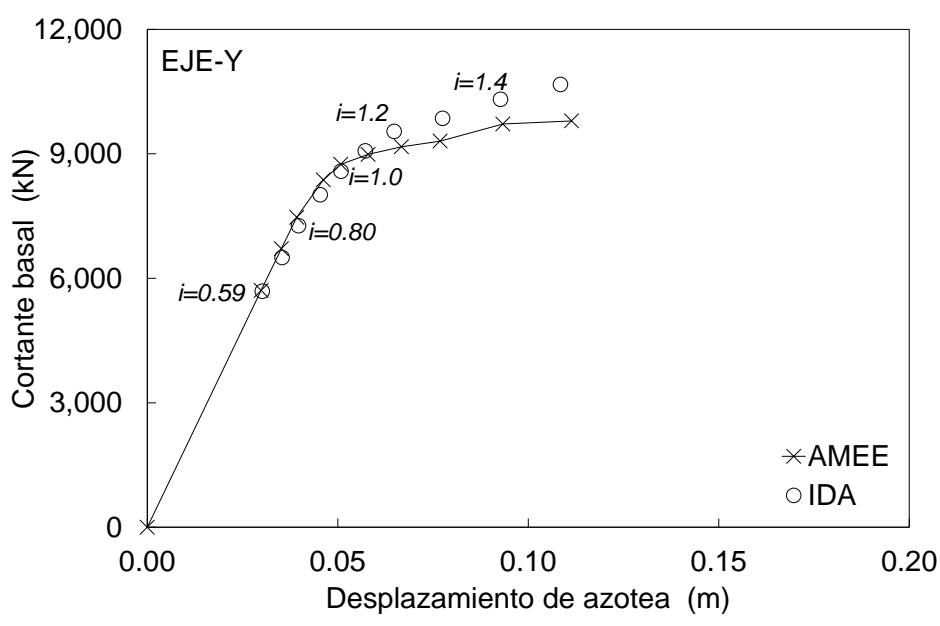

Figura 19. Curva de capacidad del modelo 8SB (dirección Y) 


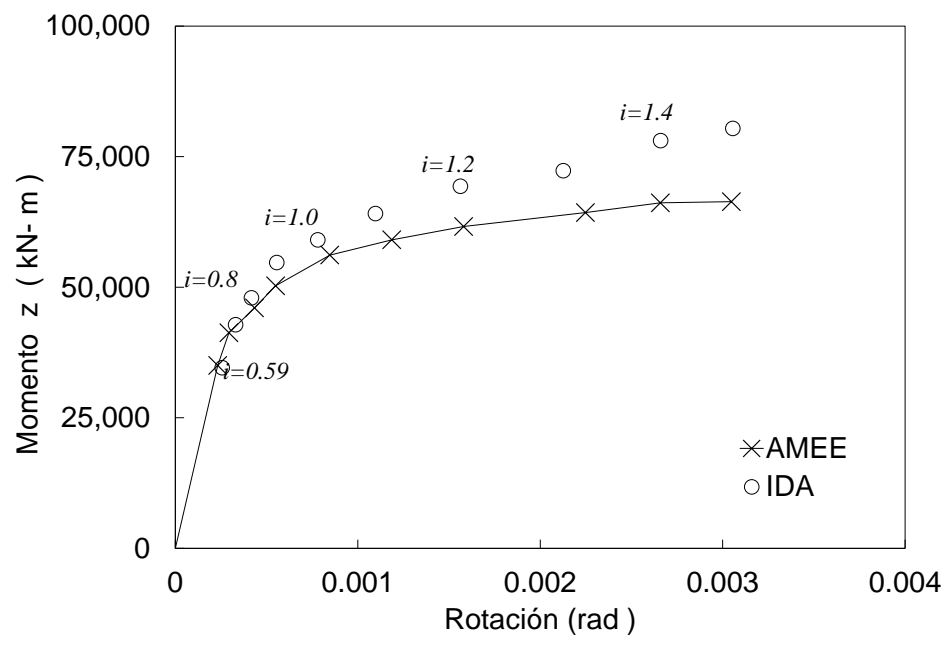

Figura 20. Curva de capacidad del modelo 8SB (dirección Rz)
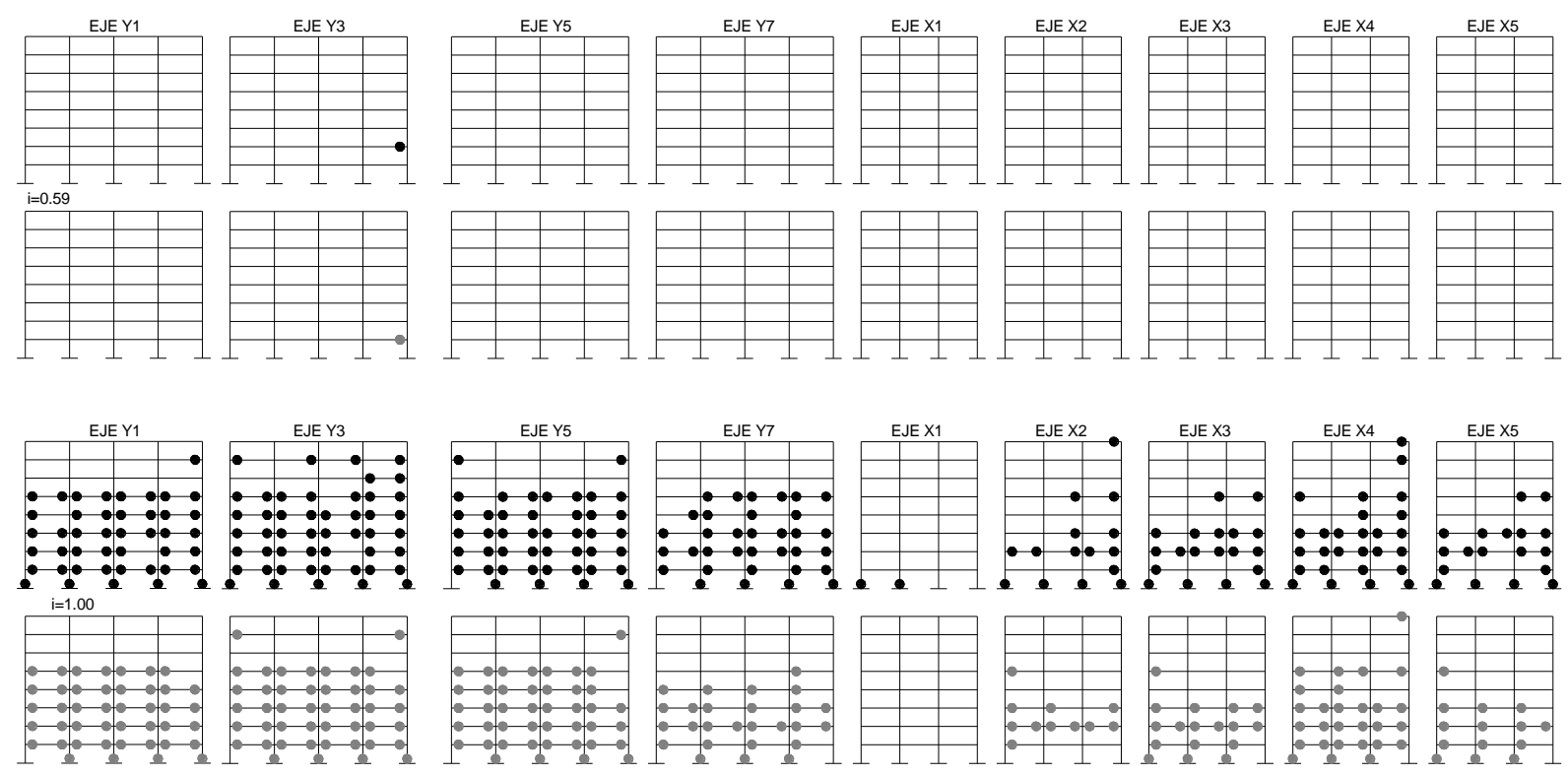

AMEE

IDA

Figura 21. Daño del modelo 8SB a diferentes intensidades de demanda sísmica

$\mathrm{Al}$ analizar las resistencias de ambas evaluaciones (8SF y $8 \mathrm{SB}$ ) se observa que son menores a las obtenidas con el IDA; esto se debe a la consideración simplista para modelar la aparición de daño, que ocasiona que una vez que el elemento estructural ha alcanzado su capacidad elástica deje de contribuir a la resistencia global de la estructura. También destaca que a medida que la intensidad de la demanda sísmica aumenta y el daño en la estructura evoluciona, se producen cambios en la configuración modal que originan pérdida de precisión en el AMEE y diferencias con los resultados de los análisis dinámicos incrementales. 
INTENSIDAD DE DEMANDA SÍSMICA $\mathrm{i}=0.59$
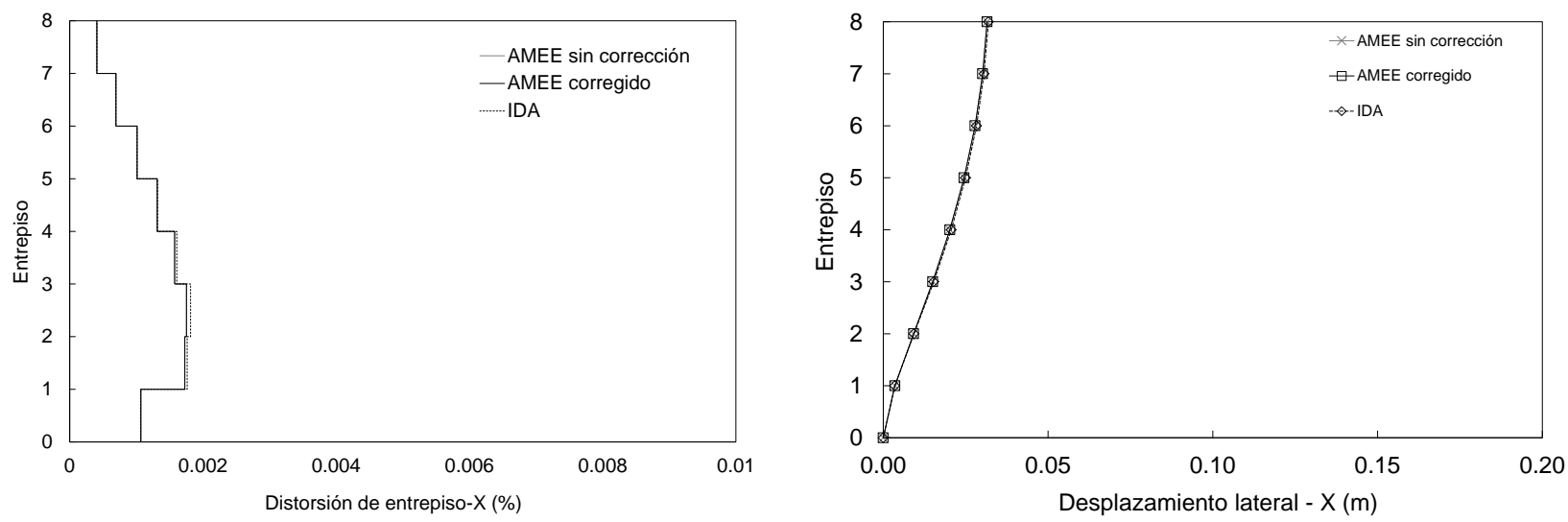

INTENSIDAD DE DEMANDA SÍSMICA $\mathrm{i}=1.00$
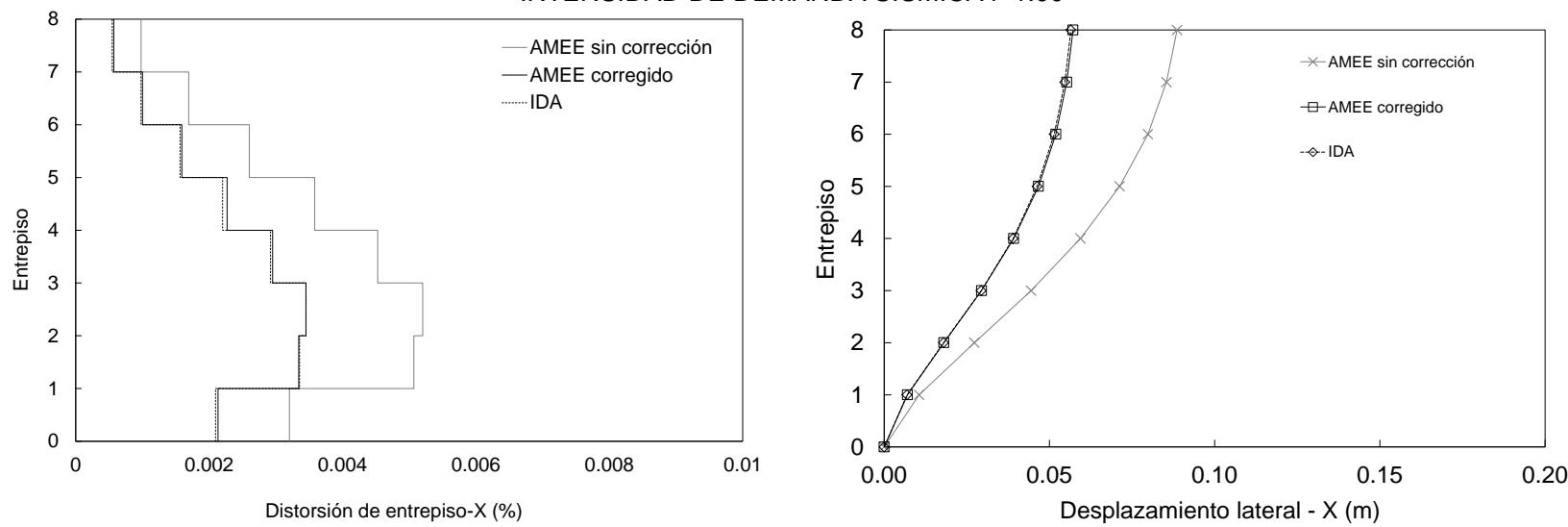

INTENSIDAD DE DEMANDA SÍSMICA $\mathrm{i}=1.50$

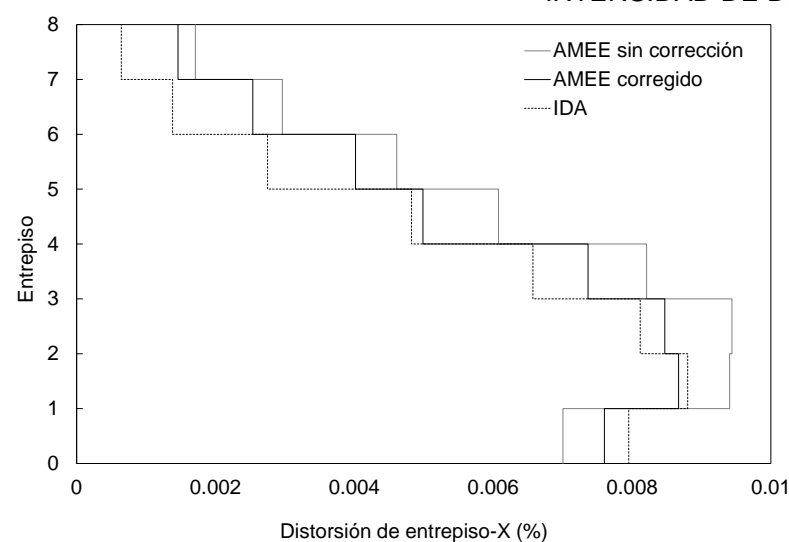

a)

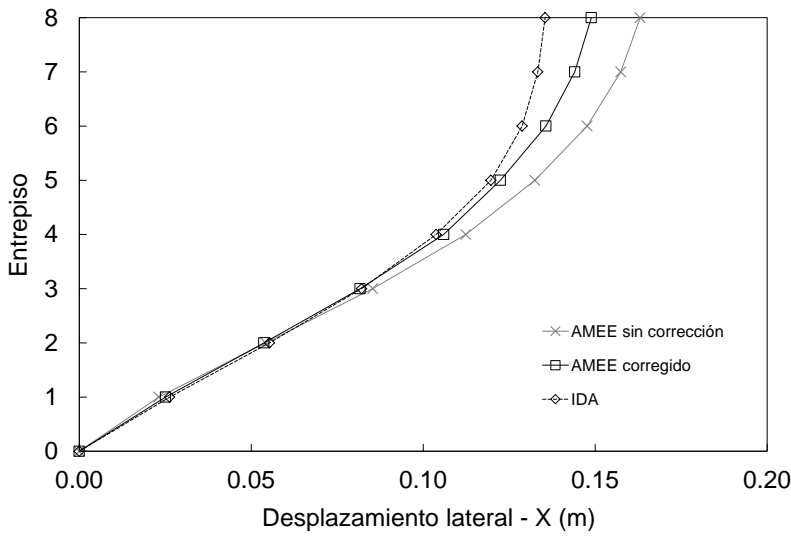

b)

Figura 22. Indicadores de desempeño global del modelo 8SB (dirección-X):

(a) Distorsiones de entrepiso y (b) Desplazamientos laterales 
INTENSIDAD DE DEMANDA SÍSMICA $\mathrm{i}=0.59$
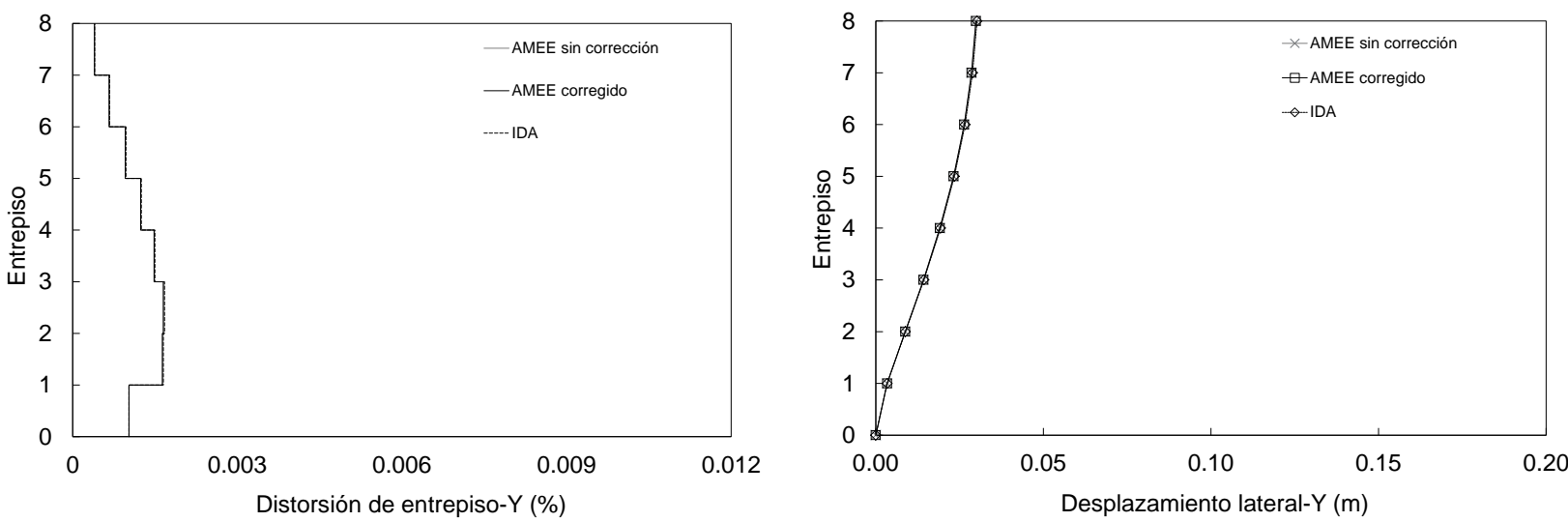

INTENSIDAD DE DEMANDA SÍSMICA $\mathrm{i}=1.00$
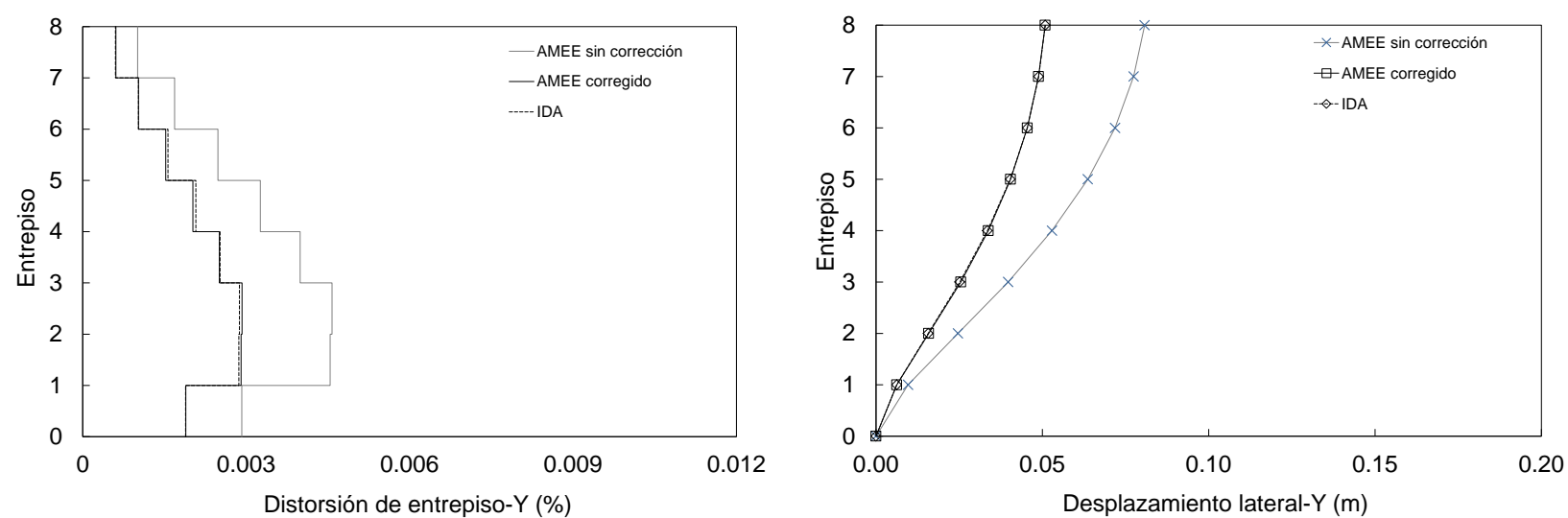

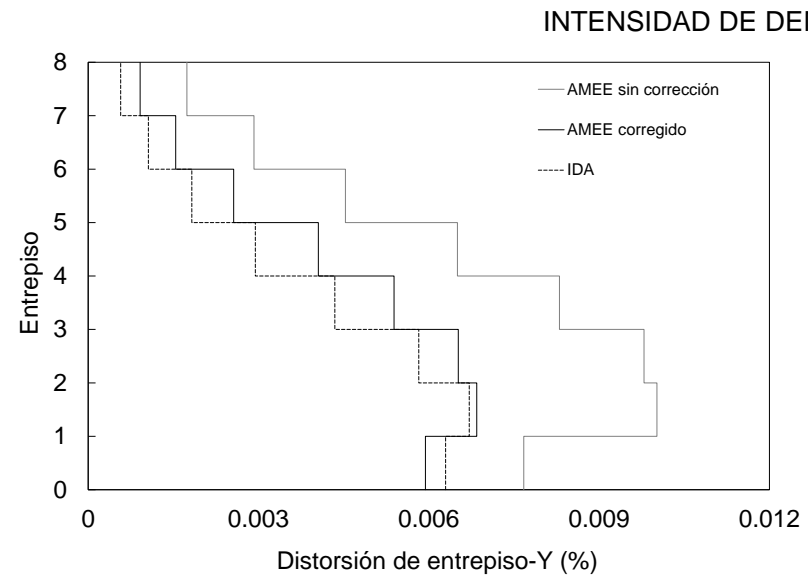

a)

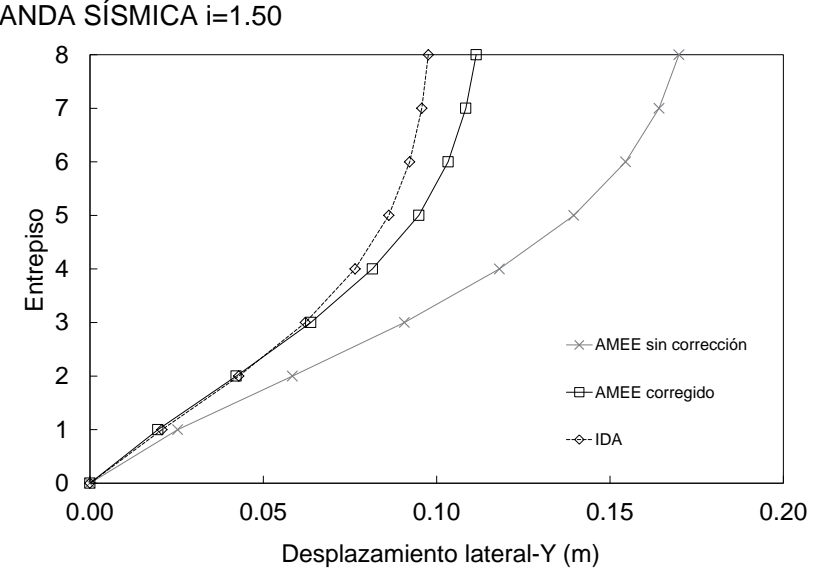

b)

Figura 23. Indicadores de desempeño global del modelo 8SB (dirección-Y):

(a) Distorsiones de entrepiso y (b) Desplazamientos laterales 


\section{CONCLUSIONES}

En este artículo se presentó un método para la evaluación sísmica aproximada de edificios de concreto reforzado. Este método se fundamenta en conceptos de ingeniería de desempeño sísmico; en considerar como una propiedad del sistema estructural a la curva de capacidad; e incluir tasas de amortiguamiento equivalente como alternativa aproximada para considerar los efectos de disipación de energía por histéresis. Para ejemplificar el potencial del método propuesto, se presentaron resultados de evaluaciones de un marco y un edificio, ambos de concreto reforzado.

Del análisis de resultados se concluye que el método de evaluación propuesto es una alternativa eficiente para estimar el desempeño global (cortante basal vs máximo desplazamiento de azotea) de estructuras de concreto reforzado. Su principal ventaja es producir curvas de capacidad (traslacionales y rotacional) que para una demanda sísmica de intensidad creciente, reflejen correctamente los desempeños globales de la estructura, correspondientes a cada una de las intensidades sísmicas consideradas. Debido a esta característica, a partir de los resultados con los que se construyen las curvas de capacidad se puede determinar el desempeño de la estructura sin necesidad de realizar acciones complementarias, como serían las que involucran la aplicación de algún método de evaluación adicional.

El uso de tasas de amortiguamiento equivalente en un sistema de un grado de libertad como opción práctica para considerar los efectos de disipación de energía por histéresis, permite comparar los desplazamientos máximos de azotea y rotaciones asociados con una intensidad de demanda sísmica determinada, con los obtenidos mediante un análisis dinámico incremental.

Finalmente no debemos olvidar que la validez de este procedimiento y de la mayoría los métodos simplificados existentes para la evaluación y el diseño sísmico de estructuras que se basan en resultados de análisis estáticos equivalentes de sistemas simplificados de un grado de libertad, pierden precisión a medida que la demanda sísmica produce en la estructura cambios en su configuración modal. Esta característica hace evidente en todos los casos usar estos procedimientos de análisis simplificados con las reservas correspondientes.

\section{AGRADECIMIENTOS}

Se agradece al Dr. Humberto Varum por los comentarios y el interés manifestado al trabajo aquí presentado. También se agradece al Consejo Nacional de Ciencia y Tecnología (CONACyT) por el patrocinio del proyecto No. 82839, "Desarrollo del marco conceptual, modelos teóricos y métodos simplificados para la evaluación y el diseño sísmico de estructuras basado en desempeño" y la beca del primer autor durante sus estudios de posgrado; además a la Dirección General de Asuntos del Personal Académico de la UNAM (DGAPA) por su patrocinio en la parte final de esta investigación, a través del proyecto No. IN121009, "Modelado numérico de problemas de daño en estructuras mediante discontinuidades interiores". Finalmente un agradecimiento especial a la Subsecretaría de Educación Superior a través del Programa de Mejoramiento al Profesorado (PROMEP) por el Apoyo de Fomento a la Generación y Aplicación innovadora del Conocimiento otorgado.

\section{REFERENCIAS}

Alba, F (2005), "Método para la evaluación del desempeño sísmico de marcos planos", Tesis de maestría, Programa de Posgrado en Ingeniería, UNAM, México. 
Antoniou, S y R Pinho (2004), "Development and verification of a displacement based adaptative pushover procedure", Journal of Earthquake Engineering, Vol. 8, No. 5, pp. 643-661.

ATC (2005), "FEMA 440, Improvement of nonlinear static seismic analysis procedures", Applied Technology Council, Redwood City, California.

Aydinoglu, M (2003), “An incremental response spectrum analysis based on inelastic spectral displacements for multi-mode seismic performance evaluation", Bulletin of Earthquake Engineering, Vol. 1, No. 1, pp. 3-36.

CSI (2000), "SAP2000, Integrate finite element analysis and design of structures", Computer and Structures Inc., Berkeley, CA.

Fragiadakis, M y D Vamvatsikos (2011), "Qualitative comparison of static pushover versus incremental dynamic analysis capacity curves", Proceedings of the 7th Hellenic National Conference on Steel Structures, Volos, Grecia.

Li, K N (1996), “CANNY-E, User's Manual, Three-dimensional nonlinear dynamic structural analysis computer program package", Canny Consultants PTE, Singapur.

Ortega, J (2001), "Efecto de la variación del periodo fundamental en la respuesta sísmica inelástica de edificios torsionalmente acoplados", Tesis de maestría, Programa de Posgrado en Ingeniería, UNAM, México.

Requena, M y G Ayala (2000), "Evaluation of a simplified method for the determination of the nonlinear seismic response of RC frames", Proceedings of the Twelfth World Conference on Earthquake Engineering, Auckland, Nueva Zelanda.

Rosenblueth, E y I Herrera (1964), "On a kind of hysteretic damping", Journal of the Engineering Mechanics Division ASCE, Vol. 90, pp. 37-48.

Vamvatsikos, D y C A Cornell (2002), "Incremental dynamic analysis", Earthquake Engineering and Structural Dynamics, Vol. 31, No. 3, pp. 491-514. 